\title{
PREDICTORS OF TREATMENT SEEKING INTENTION AMONG PEOPLE WITH COUGH IN EAST WOLLEGA, ETHIOPIA BASED ON THE THEORY OF PLANNED BEHAVIOR: A COMMUNITY BASED CROSS-SECTIONAL STUDY
}

\author{
Yohannes Addisu ${ }^{1}, Z_{\text {Zewdie Birhanu }}^{2}$, Dejen Tilahun ${ }^{2}$, Tsion Assefa ${ }^{2}$
}

\section{ABSTRACT}

BACKGROUND: Early treatment seeking for cough is crucial in the prevention and control of Tuberculosis. This study was intended to assess treatment seeking intention of people with cough of more than two weeks, and to identify its predictors.

METHODS: A community based cross-sectional study was conducted among 763 individuals with cough of more than two weeks in East Wollega Zone from March 10 to April 16, 2011. Study participants were selected from eighteen villages by cluster sampling method. Data collection instruments were developed according to the standard guideline of the theory of planned behavior. The data were analyzed with SPSS 16.0. Multiple linear regression was used to identify predictors.

RESULTS: Mean score of intention was found to be $12.6(S D=2.8)$ (range of possible score=3-15). Knowledge $(\beta=0.14,95 \% C I: 0.07-0.2)$, direct attitude $(\beta=0.31,95 \% C I: 0.25-0.35)$, belief-based attitude ( $\beta=0.03,95 \% C I: 0.02-0.06)$ and perceived subjective norm $(\beta=0.22,95 \% C I: 0.13-0.31)$ positively predicted treatment seeking intention. However, perceived behavioral control and control belief were not significantly associated with treatment seeking intention ( $p>0.05)$. Being smoker $(\beta=0.97,95 \%$ CI:-1.65$(-0.37))$ and higher family income $(\beta=0.06,95 \% C I:-0.07-(-0.01)$ were significantly associated with lower treatment seeking intention.

CONCLUSION: TPB significantly predicted treatment seeking intention among the study participants. Attitude and silent beliefs held by the respondents play an important role and should be given emphasize in prevention and control of Tuberculosis.

KEYWORDS: Tuberculosis, Cough, Intention, Treatment, Theory of Planned Behavior

\section{DOI:http://dx.doi.org/10.4314/ejhs.v24i2.5}

\section{INTRODUCTION}

Tuberculosis (TB) remains a major global health problem, as it ranks as the second leading cause of death due to infectious disease worldwide (1). Despite remarkable scientific advancements, it continues to pose considerable threat to human health (2). In 2011, there were an estimated 8.7 million new cases of $\mathrm{TB}$ and 1.4 million people died of TB. African has $24 \%$ of the world's cases and the highest rates of cases and deaths per capita (1). Ethiopia ranks $7^{\text {th }}$ out of the world's twentytwo high burden countries for TB (3). According to the 2009 estimates, Ethiopia is a high TB and MDR-TB country with estimated incidence, prevalence and mortality rates of $359 / 100,000,572 / 100,000$ and 64/100,000 respectively (3). As indicated in the health related

${ }^{1}$ Department of Public Health, Dilla University, Ethiopia

${ }^{2}$ Department of Health Education \& Behavioral Sciences, College of Public Health \& Medical Sciences, Jimma University, Ethiopia Corresponding Author: Zewdie Birhanu, Email: zbkoricha@y ahoo.com 
report of 2009, tuberculosis was the sixth cause of hospital admission (4).

In Ethiopia, the proportion of tuberculosis cases detected and cured under DOTs are far behind the targets (5). The case detection rate of $70 \%$, as targeted by WHO, may not be achieved as it now stands at only $34 \%$ (6). In Oromia Region, case detection rate was $36 \%$ in 2009(5). This indicates that very few TB patients visit health facilities while a vast majority of the patients delayed from treatment and remain in community which in turn scales up the current burden of tuberculosis (6, 7). Early treatment seeking behavior has a paramount role to prevent death from TB; prevent relapse; decrease transmission and prevent the development of drug resistance TB $(8,9)$. The cycle of the disease transmission will continue in the community and make TB the most challenging human health problem (9). Thus, to curb the burden of $\mathrm{TB}$, it is recommended that anyone with cough of more than two weeks should visit health care facility for check-up of TB (5). However, people's intention of seeking early treatment in the experience of cough has not yet achieved in Ethiopia. Therefore, this study was intended to measure treatment seeking intention and associated factors among people with cough of more than two weeks.

This study used the Theory of Planned Behavior (TPB) as a conceptual framework. TPB asserts that intention is the most immediate predictor of behaviors which in turn is influenced by attitude towards the behavior, subjective norms and Perceived Behavioral Control (PBC) $(10,11)$. Subjective norm is the individual's perception of the likelihood that the potential referent group or individuals approve or disapprove of performing the given behavior $(10,11)$. The rationale to this, under significant social influence and social pressure, an individual would perform the behavior even though he/she is not in favor of performing the behavior (10). PBC is the individual's perception of the ease or difficulty in performing the behavior of interest (11), seeking treatment in this case. The performance of a particular behavior is correlated with the confidence of the individual in his/her ability of performing the behavior $(10,11)$. Similarly, according to the TPB, attitude is the evaluative effect of positive or negative feeling of individuals in performing a particular behavior (10).

\section{METHODS AND MATERIALS}

A community based cross-sectional study design was conducted among people with cough of more than two weeks in Sibu Sire District, East Wollega Zone of Oromia National Regional State from March 10 to April 16, 2011. Located $281 \mathrm{Kms}$ away from Addis Ababa (the capital of Ethiopia), Sibu Sire District consists of 19 rural Gandas (smallest administrative units in Oromiya) and three semi-urban Gandas. TB diagnosis service and DOTs program were being given only at one health center in the district. However, DOTs service was being provided at five health posts.

Respondents were included in the study if they were older than 15 years and had a cough of more than two weeks. Those who were on DOTs and/or visited health care facility but did not get treatment were excluded from the study.

The sample size was calculated assuming p, proportion of people with cough of more than two weeks who intended to seek treatment, to be $50 \%$. This proportion was preferred due to lack of similar studies in Ethiopia. Other assumptions made were $5 \%$ marginal error (d) and confidence interval of $95 \%$. Based on these assumptions, the sample size was calculated using a formula $n=\left(Z_{1}\right.$. $\dot{\alpha} / 2)^{2} \mathrm{p}(1-\mathrm{p}) / \mathrm{d}^{2}$. This gives a sample size of 384 . To correct for the design effect, the sample size was multiplied by a factor of two. Assuming $10 \%$ nonresponse rate, the final sample size was 845 respondents.

Respondents were sampled as follows: First, Gandas, which belong to Sibu Sire District, were stratified into rural and semi-urban. Then, sixteen rural Gandas and two semi-urban Gandas were randomly selected. The sample size was proportionally allocated to each selected Ganda based on the total number of households. Households residing in all of the selected Gandas were surveyed to recruit respondents who had a cough of more than two weeks. Self-report method was used to assess the duration of cough, and a respondent was included if his/her cough persisted for longer than two weeks.

Research instruments were developed according to the guideline of the TPB. First, open-ended elicitation interviews were conducted among 15 respondents to identify salient belief underlying attitude, subjective norm and perceived behavioral control. Then, the data were analyzed 
and used to develop quantitative questionnaires. Accordingly, 9 items on behavioral belief, 12 items on normative beliefs and 8 items on control beliefs were obtained. These questionnaires were translated into Afan Oromo and pre-tested. Exploratory factor analysis with varmax rotation method was done to determine whether items tapped into the same construct. Only factors with Eigen value of $\geq 1$ were retained for further analysis. Regarding reliability, Cronbach's Alpha ranged from 0.76 to 0.92 .

Intention to seek treatment (I): Intention to seek treatment is the outcome variable in this study. It is the motivation in the sense of one's conscious plan to perform a given behavior (10). Three items were presented to describe respondents' level of agreement in a five scale response format ranging from "strongly disagree" to "strongly agree". Then, these items were summed up to produce intention score and the score used in regression analysis.

Attitude towards TB treatment: Attitude was measured in two ways-by asking the respondents' direct degree of favor or disfavor through four items presented in five-point semantic differential scales. Items of behavioral beliefs [four items] and outcome evaluation [four items] were used to compose the attitude scale where each behavioral beliefs item scores were multiplied by its corresponding outcome evaluation item scores and then summed up to compose the belief based attitude scale.

Subjective norm towards treatment seeking (SN): Subjective norm was measured in two ways. Three items were used to measure the subjective norm directly; the respondents rated their level of agreement or disagreement with five category Likert scale measures. Items of normative beliefs [four items] and motivation to comply [four items] were used to compose the subjective norm scale where the score of each normative belief item was multiplied by its corresponding motivation to comply item, and summed up to compose the belief based subjective norm scale.

Perceived behavioral controls to seek treatment (PBC): Likewise, the PBC was measured in two ways; directly with four items on five point Likert scale category. Indirectly, items of control beliefs [four items] and power of control [four items] were used to compose the belief based perceived behavioral control.

Distal variables included socio-demographic characteristics and knowledge about TB. Knowledge was assessed using eight items scored as " 1 " for a correct answer, and " 0 " for an incorrect answer. Then, the items were summed up to produce composite measure index which was used for regression analysis.

The data were collected by trained individuals who completed grade ten. Data were analyzed by SPSS.16.0. Descriptive statistics were used to summarize the findings. Pearson correlation was computed to determine the correlation of direct measures of TPB variables with their corresponding indirect measures and intention. Stepwise multiple linear regressions were used to identify variables which independently contributed to the prediction of treatment seeking intention. First, the effect of direct measures of the TPB was examined followed by evaluation of the effect of indirect measures. Secondly, the effects of both direct and indirect measures were examined to determine the predictive power of the TPB. The final regression model was developed based on stepwise regression method with $95 \%$ Confidence Interval (CI) and significance level set at less than 0.05.

This study was approved by the Ethical Clearance Committee of Jimma University; verbal informed consent was obtained from each respondent.

\section{RESULTS}

Socio-demographic characteristics of the respondents: A total of 763 respondents participated in the study giving a response rate of $90.4 \%$. The mean age of respondents was 36.90 years $(\mathrm{SD}=15.11)$. Six-hundred and fifty-nine $(86.4 \%)$ of the respondents were recruited from rural Gandas. Regarding marital status, $540(70.8 \%)$ and $39(5.1 \%)$ of the respondents were married and widowed, respectively. In terms of religion, 359(47.1\%) of them were protestants followed by Orthodox Christianity who accounted for $316(41.4 \%)$ of the total. The majority of the respondents, 649(85.1\%), were Oromos followed by Amharas which accounted for 98(12.8\%). Most of the respondents, 698(91.5\%), attended primary education. 
Knowledge about TB and its prevention methods: Five hundred thirty Seven $(70.4 \%)$ of the respondents believed that $\mathrm{TB}$ is caused by infectious agents called TB germs, and $444(30.3 \%)$ of them knew that TB is a contiguous disease. Belief about transmission showed that for $568(74.4 \%)$ of the respondents, TB is a transmittable disease and for $578(75.8 \%)$ of them, it is curable. Four hundred Seven $(53.3 \%)$ of the respondents knew that TB is transmitted through coughing from TB patients.

More than half of the respondents, 408 $(53.5 \%)$, knew that lack of enough ventilation can transmit TB. However, $385(50.5 \%)$ of them believed that sunlight cannot kill TB germs.
Treatment seeking intention for cough: Table 1 presents respondents' intention to seek treatment for cough. Accordingly, the mean score of intention items was $12.6(\mathrm{SD}=2.8)$ (range of possible score $=3-15$ ) implying that the majority of the respondents had intention to visit health facility to get remedy for their illness. A close look into each item of the scale also indicates that many respondents intended to visit health institutions in the near future. For instance, in response to item: "I intend to go to health facility and have a check up for TB", $638(83.6 \%)$ agreed with the statement and only $73(9.6 \%)$ disagreed.

Table 1: Treatment seeking intention for cough, Sibu Sire District, May 2011

\begin{tabular}{llll}
\hline \multicolumn{1}{c}{ Items } & $\begin{array}{l}\text { Agree } \\
\mathrm{No}(\%)\end{array}$ & $\begin{array}{l}\text { Disagree } \\
\mathrm{No}(\%)\end{array}$ & $\begin{array}{l}\text { Not sure } \\
\mathrm{No}(\%)\end{array}$ \\
\hline $\begin{array}{l}\text { I expect people with cough of more } \\
\text { than 2weeks should visit health } \\
\text { facility }\end{array}$ & $36(47.4)$ & $351(46.0)$ & $50(6.6)$ \\
$\begin{array}{l}\text { I want to go to health facility and have } \\
\text { a check up for TB }\end{array}$ & $647(84.8)$ & $74(9.7)$ & $42(5.5)$ \\
$\begin{array}{l}\text { I intend to go to health facility and } \\
\text { have a check up for TB } \\
\text { Mean=12.6 } \pm 2.8\end{array}$ & & $73(9.6)$ & $52(6.8)$ \\
\hline
\end{tabular}

Correlation of direct measures of TPB variables with treatment seeking intention: The mean score to direct measures of TPB variables were $12.9(\mathrm{SD}=2.8)$ for attitude (range of possible score $=4-20), 7.8 \quad(\mathrm{SD}=2.0) \quad$ (range of possible score $=3-15)$ for subjective norm and 11.3 $(\mathrm{SD}=3.6)$ (range of possible score $=4-20$ ) for perceived behavioral control. Pearson correlation analysis indicated that all the direct measures were significantly correlated with treatment seeking intention: attitude $(\mathrm{r}=0.54, \mathrm{p}=0.001) \mathrm{PBC}(\mathrm{r}=0.31$, $\mathrm{p}=0.001)$, and subjective norm $(\mathrm{r}=0.42, \mathrm{p}=0.001)$.

Predicting treatment seeking intention from indirect measures of TPB variables: Consistent with the expectations based on the TPB, the indirect measure of subjective norm (weighted normative belief) was significantly correlated with the direct measure of subjective norm( $(\mathrm{r}=0.67)$. However, the indirect measure of attitude (weighted behavioral beliefs) $(\mathrm{r}=0.27)$ and perceived behavioral control (weighted control beliefs) $(r=0.25)$ had weak correlation with the direct measure of attitude and perceived behavioral control. This could suggest that direct and indirect measures of attitude and behavioral control predictors may assess different psychological constructs which independently contribute to the prediction of intention. Therefore, prediction of treatment seeking intention may be improved by treating these as separate predictors. Table 2 shows the effect of behavioral beliefs, normative beliefs and control belief on treatment seeking intention. All of these scales were independently significantly contributed for the prediction of treatment seeking intention $(\mathrm{p}<0.05)$. 
Table 2: Predicting treatment seeking intention from indirect measures of TPB, May 2011

\begin{tabular}{llll}
\hline Indirect measures & Beta & Sig & 95\%CI for Beta \\
\hline weighted behavioral belief & 0.340 & 0.001 & $0.030-0.050$ \\
Weighted normative belief & 0.191 & 0.001 & $0.013-0.034$ \\
weighted control beliefs & 0.237 & 0.001 & $0.014-0.023$ \\
\hline
\end{tabular}

Relationship between distal variables and treatment seeking intention: It was found out that only residence $(\beta=0.43, \mathrm{P}=0.001)$, being smoker $(\beta=-0.098, p=0.002)$, monthly income $(\beta=-$
$0.12, p=0.001)$ and knowledge $(\beta=0.23, P=0.001)$ significantly and independently predicted treatment seeking intention (Table 3).

Table 3: Predicting treatment seeking intention from distal variables, May 2011

\begin{tabular}{lccc}
\hline Distal variables & Beta coefficients & Sig. & 95\% CI for B \\
\hline Residence (urban/rural*) & 0.430 & 0.001 & $2.99-3.97$ \\
family monthly income & -0.118 & 0.001 & $-0.01-0.23$ \\
Smoking (yes/no*) & -0.096 & 0.002 & $-2.02-0-.47$ \\
Knowledge & 0.231 & 0.001 & $0.23-0.38$ \\
\hline
\end{tabular}

*reference category

Pre dictors of tre atment seeking intention: The effect of distal and TPB variables on treatment seeking intention was evaluated, and the result is presented in table 4. In this stepwise regression model, only variables which were significantly associated with treatment seeking intention in the previous models are included.

First, distal variables were regressed upon intention and found $\mathrm{R}^{2}=0.2950(\mathrm{p}<0.001)$. This indicates $29.50 \%$ of treatment seeking intention was explained by these distal variables. Of these distal variables, residence and smoking status had major effects on treatment seeking intention: living in urban areas $(\beta=1.97,95 \% \mathrm{CI}$ : $1.56-2.39$, $\mathrm{p}=0.001)$ and being smoker $(\beta=-0.97,95 \% \mathrm{CI}$,-
1.65 to $-0.37 \mathrm{p}=0.002$ ) and were significantly associated with treatment seeking intention. Then, constructs of TPB were regressed upon intention and resulted in $\mathrm{R}^{2}=0.579$, suggesting that $28.40 \%$ of the variability in treatment seeking intention was explained by the constructs of TPB. The analysis revealed that direct attitude had a huge effect on treatment seeking intention $(\beta=0.31$, 95\% CI: $0.25-0.35, p=0.001)$, and was followed by perceived subjective norm $(\beta=0.22,95 \% \mathrm{CI}$ : $0.13-0.31, \mathrm{p}=0.001)$. Among the constructs of TPB, direct attitude (variance $=17.00 \%$ ) and perceived subjective norm (variance $=7.80 \%$ ) had considerable effects on treatment seeking intention. 
Vol. 24, No. 2

April 2014

Table 4: Predictors of treatment seeking intention, May 2011

\begin{tabular}{llcc}
\hline Predictor variables & Unstandardized Beta & Sig. & 95\% CI \\
\hline Residence (urban/rural*) & $1.97(\mathrm{SD}=0.21)$ & 0.001 & $1.56-2.39$ \\
Family monthly income & $-0.06(\mathrm{SD}=0.01)$ & 0.001 & $-0.07--0.01$ \\
Smoking (yes/no*) & $-0.97(\mathrm{SD}=0.30)$ & 0.002 & $-1.65--0.37$ \\
Knowledge & $0.14(\mathrm{SD}=0.03)$ & 0.001 & $0.07-0.20$ \\
Direct attitude & $0.31(\mathrm{SD}=0.02)$ & 0.001 & $0.25-0.35$ \\
Belief based attitude & $0.03(\mathrm{SD}=0.01)$ & 0.001 & $0.02-0.06$ \\
Perceived subjective norm & $0.22(\mathrm{SD}=0.04)$ & 0.001 & $0.13-0.31$ \\
Perceived behavioral control & $0.06(\mathrm{SD}=0.03)$ & 0.094 & $0.07-1.13$ \\
Control belief & $-0.01(\mathrm{SD}=0.01)$ & 0.517 & $-0.01-0.07$ \\
\hline
\end{tabular}

*reference category

\section{DISCUSSION}

The present study attempted to identify predictors of treatment seeking intention among people with cough of more than two weeks. The study showed that distal variables explained $29.50 \%$ of treatment seeking intention whereas the constructs of TPB together explained $28.40 \%$ of the variance. In sum, $57.90 \%$ of the variability in treatment seeking intention was explained by the conceptual framework as a whole. In this study, distal variables including place of residence, being smoker, family monthly income and knowledge about TB were significant predictors of treatment seeking intention which is also documented in some previous studies $(7,12)$. An individual's ability to recall or know about the etiology of a disease, chain of disease process, symptoms, preventions and treatment options plays a crucial to motivate him/her to seek treatment (13). Consistent with pervious study in Ethiopia (14), knowledge is a significant predictor of intention to seek treatment in this study.

Of the TPB constructs, attitude plays a key role to determining treatment seeking intention as it explained the largest variability which was also reported in previous studies (15-19). In addition, the established attitude-intention relationship in the current study reflected the underlying assumption of the theory of planned behaviors which states that the more favorable the attitude towards the behavior, the stronger should be the individual's intention to perform it (20). Similarly, subjective norm has a positive and significant effect on treatment seeking intention, and explained $1.3 \%$ of the variability. However, literatures have shown mixed results regarding subjective norm as a predictor of intention. While some studies have shown no significant relationship between subjective norm and intention (21), other studies have revealed significant relationship $(8,15)$. This could be due to the fact that respondents are different in terms of socio-cultural factors and sources of social pressures. In addition, the salient belief measures due to nature of TPB, which is local and culture sensitive, might also contributed to the difference between the findings. Moreover, the inconsistencies of the findings may be due to the measurement method: many authors use single item measures (21) as opposed to more reliable multi-item scales.

Studies have also shown that perceived behavioral control accounted for considerable variance in intention and behavior. As a result, there is a positive relationship between perceived behavioral control and intention $(5,15,16,17$, 22). Thus, with the given resources, the higher the confidence in individual's ability, the more likely he/she visits health care facility. However, the current study revealed that there was no statistically significant relation between perceived behavioral control and treatment seeking intention. This implies that seeking treatment for cough is under volitional control of the individuals in this study and perceived behavioral control should not exert any influence on intention-behavior relationships. Thus, the implementation of an intention into action in this case is less likely to be determined by environmental barriers. The lack of evidence for the interactive effects of perceived behavioral control on the intention might be partly 
explained by the fact that the relative importance of attitude, subjective norm and perceived behavioral control in the prediction of intention varies across behaviors and situations (23). That is, in situations where attitudes are strong predictors as in the present study, or where normative influences are powerful, perceived behavioral control may be less predictive of intentions. Ajzen also argues that the magnitude of the perceived behavioral control-intention relationship is dependent upon the type of behavior and the nature of the situation $(9,23)$.

In conclusion, the present study revealed that there was a high intention to seek treatment for cough. Distal variables and constructs of the TPB explained nearly equal variance in treatment seeking intention. It suggested that both categories of variables are equally important and should be taken into account in behavioral change communication. Attitude towards seeking treatment and perceived social pressure are highly relevant to promotion of early treatment seeking behavior, whereas perceived behavioral control is less relevant. This implies that treatment seeking behavior for cough is relatively under personal control, and is less likely to be affected by environmental barriers. As this study is the first of its kind in Ethiopia, it has an important implication for TB prevention and control program in such a limited-resource setting. Thus, practitioners should focus on changing negative attitude, lessening the impact of social pressures, dispelling negative evaluation and salient beliefs underlying attitude. Further studies can be required to establish the intention-behavior link in this particular population and settings. However, the fact that the theory of planned behaviors is purely psychological and lack of similar local literatures may limit the scope of this study.

\section{ACKNOWLEDGEMENTS}

We acknowledge Jimma University for its financial assistance. We are also grateful to the respondents of this study for their participation. In addition, local administrators in the study sites deserve acknowledgement for their unreserved cooperations.

\section{REFERENCES}

1. World Health Organization: Global Tuberclosis Report: WHO Library Cataloguing-in-Publication Data. Geneva, 2012.

2. World Health Organization. Global tuberculosis control: epidemiology, strategy, is financing. WHO report 2009.

3. $\mathrm{WHO} / \mathrm{CCO} /$ Ethiopia. Country cooperation strategy at glance. May 2011.

4. FMOH/Ethiopia. Health and health related indicator. Addis Ababa 2008/2009.

5. Federal Ministry of Health of Ethiopia. Implementation Guidelines for TB/HIV Collaborative Activities in Ethiopia. Addis Ababa 2007.

6. Zaman, k, Tuberculosis. A global health problem. Journal of health, population and nutrition 2010; 28 (2).

7. Ayelew T A, McommH, Meseret Y. Delays in tuberculosis treatment and associated factors in Jimma Zone, Southwest Ethiopia. Ethiop J Health Sci, 2009; 19 (1):29-37.

8. Jimma University. In Proceeding of the fifth National TB workshop, Organized by Tuberculosis research Advisory Committee (TRAC). Jimma 2009.

9. WHO: Global TB control: WHO report. Geneva 2011.

10. Ajzen I. Perceived behavioral control, selfefficacy, locus of control and the theory of planned behavior. Journal of Applied Social Psychology, 200; 32:1-20.

11. Fishbein M, Ajzen I. Belief, Attitude, Intention, and Behavior: An Introduction to Theory and Research. Addison-Wesley, Reading 1975.

12. Thaw M. Patient delay in tuberculosis center treatment among Mayanmer migrants, Samutsskhon province. Thailand 2005 (unpublished).

13. Rumman A, Sabra N, Bakri F, Seita A, Bassili A. Prevalence of tuberculosis suspects and their healthcare-seeking behavior in urban and rural Jordan. The American journal of tropical medicine and hygiene, 2008; 79(4):545-51.

14. Solomon Y, Hansen C, Yimaldu T, Bjune G. Health care seeking among pulmonary TB 
suspect \& patient in rural Ethiopia. $B M C$ public health, 2009; 9-454.

15. Henari, JE Golub, CI, Mohan, GW Comstock, RE Chasisson. Active case finding of Tuberculosis: historical perspective and further aspects. Int J Tuberc lung dis, 2009; 9(11):1183-203.

16. Goehaman SD. Health Behavior: emerging research perspectives. Raymond S. Kent School of Social Work, University of Louisville 1998.

17. Murray CJ, Styblo K, Rouillon A. Tuberculosis in developing countries: burden, intervention and cost. Bull Int Union Tuberc Lung Dis, 1990; 65(1):6-24.

18. Donald R, van Helden P. The Global Burden of Tuberculosis: Combating Drug Resistance in Difficult Times. N Engl J Med, 2009; 360:2393-2395.
19. WHO. Global Disease Burden measured in Disability: Adjusted Life Year (DALYs). WHO health report 2004; 1:75.

20. Christopher J, Armitage M. Efficacy of the Theory of Planned Behaviour: A metaanalytic review. British Journal of Social Psychology, 2001; 40(4): 471-499.

21. Joyce B, Jane H. Medical-Surgical Nursing: A Clinical Management for positive outcomes. Seventh Edition, Elsevier: Sounders, 2005.

22. WHO Stop TB partnership. Fact sheet global plan to stop TB 2011 - 2015: transforming the fight towards elimination of Tuberculosis. Geneva 2011.

23. Ajzen I. The theory of planned behavior. Organizational Behavior and Human Decision Processes, 1991; 50(2): 179-211. 